\title{
The patterns and timing of recurrence after curative resection for gastric cancer in China
}

\author{
Dan Liu', Ming Lu', Jian Li ${ }^{1}$, Zuyao Yang ${ }^{2}$, Qi Feng ${ }^{2}$, Menglong Zhou ${ }^{3}$, Zhen Zhang ${ }^{3^{*}}$ and Lin Shen ${ }^{1 *}$
}

\begin{abstract}
Background: The recurrence of gastric cancer after curative resection had adverse effects on patients' survival. The treatment presence varied from different countries. The aims of this study were to understand the recurrence incidence, patterns, and timing and to explore the risk factors in China.

Methods: One thousand three hundred four patients who undergoing curative resection from more than 100 hospitals between January 1st 1986 and September 1st 2013, were surveyed in detail. Clinical pathological factors were examined as potential risk factors of each recurrence pattern using univariate and multivariate analyses. Recurrence timing was also analyzed based on disease-free survival.

Results: Among 1304 gastric cancer patients, 793 patients (60.8\%) experienced recurrence and 554 patients (42.5\%) experienced recurrence within 2 years after operation. The median disease-free survival was 29.00 months (interquartile range $[\mathrm{IQR}] 12.07,147.23)$. Receiving operation in general hospitals was one of independent risk factors of local-regional recurrence $(\mathrm{OR}=1.724,95 \% \mathrm{Cl} 1.312$ to 2.265$)$ and distant metastasis $(\mathrm{OR}=1.496,95 \% \mathrm{Cl} 1$. 164 to 1.940). Patients would suffer lower risk of distant metastasis if they received no more than 3 cycles adjuvant chemotherapy ( $\mathrm{OR}=0.640,95 \% \mathrm{Cl} 0.433$ to 0.943 ). Adjuvant radiotherapy could reduce the risk of recurrence (OR 0 . $259,95 \% \mathrm{Cl} 0.100$ to 0.670 ), especially distant metastasis ( $\mathrm{OR}=0.260,95 \% \mathrm{Cl} 0.083$ to 0.816 ).

Conclusions: More than $60 \%$ patients experienced recurrence after curative resection for gastric cancer, especially within 2 years after surgery. Risk factors were clarified between various recurrence patterns. Advanced gastric cancer and undergoing operation in general hospitals contributed to increased recurrence risk and worse survival. Enough number of lymph nodes harvest and standard D2 lymphadenectomy could reduce recurrence. Chinese patients would benefit from adjuvant chemotherapy and radiotherapy.
\end{abstract}

Keywords: Gastric cancer, Curative resection, Postoperative recurrence, Disease-free survival, Overall survival

\section{Background}

Gastric cancer is the fourth common malignant tumor in the world, and China is one of countries with high gastric cancer incidence [1,2]. So far, curative resection has been considered as the only way to cure gastric cancer. Recurrence after curative resection contributes

\footnotetext{
* Correspondence: zhenzhang6@gmail.com; linshenpku@163.com ${ }^{3}$ Department of Radiation Oncology, Shanghai Cancer Center, Fudan University, Shanghai 200032, China

${ }^{1}$ Key Laboratory of Carcinogenesis and Translational Research (Ministry of Education), Department of Gastrointestinal Oncology, Peking University Cancer Hospital and Institute, No. 52, Fucheng Road, Haidian District, Beijing 100142, China

Full list of author information is available at the end of the article
}

to the limited survival of patients. Recurrence patterns generally include local-regional recurrence, distant or hematogenous metastasis, and peritoneum implanting. Recurrence patterns have related to adjuvant treatment modes. For example, in America, local-regional recurrence and hematogenous metastasis were fairly common, and patients could benefit from adjuvant radiochemotherapy [3-5]. While, in Japan and South Korea, where distant metastasis and peritoneum implanting were regular, patients could benefit from adjuvant chemotherapy, rather than adjuvant radio-chemotherapy [6-9]. Moreover, recurrence timing could provide information of postoperative follow-up, in order to find 
recurrence timely. In China, several small sample singlecenter studies had reported the recurrence patterns and relative risk factors [10-12], but little large sample and multiple centers analysis had reported recurrence timing and survival. And the level of gastric cancer treatment varied from different hospitals, sites, and times. So, that exploring the actual recurrence patterns and timing was necessary. It was the first large sample of multiplecenter retrospective analysis for recurrence patterns and timing after curative gastric cancer resection in China. We specifically analyzed the relationship between surgical hospitals and recurrence. It would provide more information for clinicians in choosing individual treatment schedules and predicting prognosis.

\section{Methods}

\section{Patients}

There were 1646 patients, who underwent operation for gastric malignant tumors between January 1st 1986 and September 1st 2013, in more than 100 Chinese hospitals, screened for this analysis. All of them finally received postoperative treatment in Gastrointestinal Tumor Department of Beijing Cancer Hospital and Radiotherapy Department of Fudan University Shanghai Cancer Center. Three hundred forty-two patients were excluded for the following reasons: (1) 192 patients underwent palliative tumor resection (including both primary and non-local regional metastasis lesions totally resected) or experienced microscopically/visible positive (R1/R2) margin status, (2) 67 patients whose postoperative histological examinations turned out not to be gastric adenocarcinoma, and (3) 83 patients experienced death whose recurrence time and patterns were unclear. Finally, 1304 gastric adenocarcinoma patients were included in this study for recurrence patterns and timing after curative resection (Fig. 1).

\section{Data collection}

All patients' clinical pathological characteristics, including age, sex, tumor location, surgical hospitals (general and specialist hospitals), depth of tumor invasion, number of positive lymph nodes, extent of lymphadenectomy, histological type, neo-adjuvant treatment, adjuvant treatment, and recurrence and survival information were retrospectively reviewed based on operative notes and medical records.

\section{Pathological identification}

Cardia and fundus tumors were identified as gastroesophageal junction (GEJ) adenocarcinoma. Tumors located in the rest sites of gastric were identified as nongastroesophageal junction (non-GEJ) adenocarcinoma. The lymphadenectomy extent was defined according to the 2010 Japanese gastric cancer treatment guidelines [13]. The tumors' stages were classified based on the postoperative tissues, according to the 7th edition American Joint Committee on Cancer (AJCC) staging system of gastric adenocarcinoma. Histological classification was in accordance with the World Health Organization (WHO) classification of tumors of the digestive system [14]. Well-differentiated tumors included highly and moderately differentiated papillary carcinoma. And poorly differentiated included low differentiated papillary carcinoma, mucinous adenocarcinoma, and hepatoid adenocarcinoma. Recurrence was defined as biopsy and imaging highly suspicious of recurrence. Recurrence patterns included local-regional recurrence (gastric or nodal), distant metastasis (organs and distant lymph nodes), and peritoneum implanting (peritoneum nodules, ascites, and Krukenberg tumors).

\section{Postoperative follow-up}

All patients were followed from the date of surgery to death or emigration. The last follow-up of all recurrence

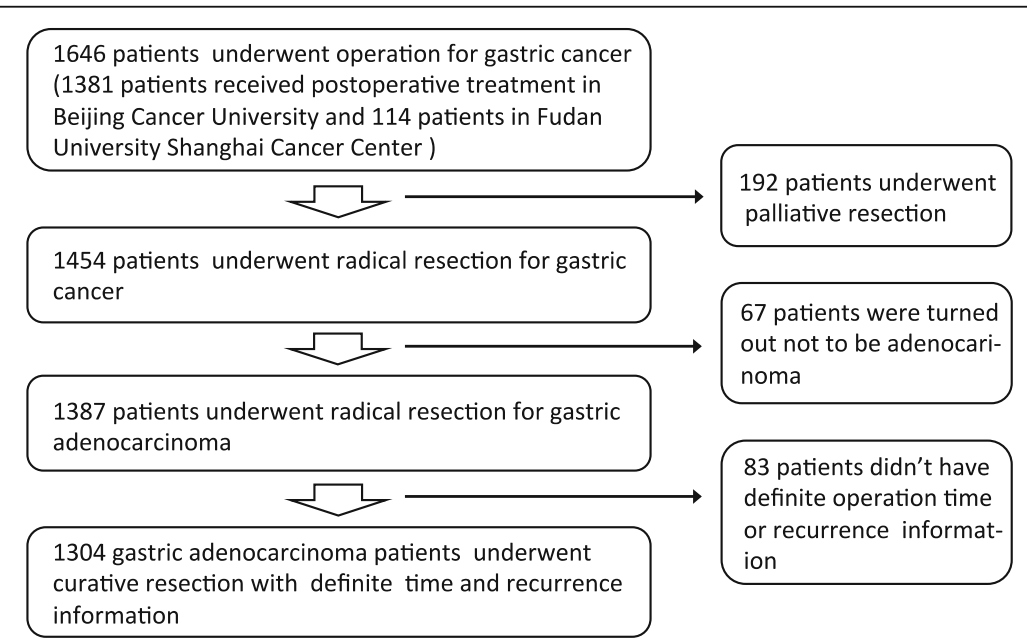

Fig. 1 The screening progress for analysis patients 
Table 1 The clinical pathological characteristics of patents

\begin{tabular}{|c|c|}
\hline Clinical features & No. of patients $(n=1304)$ \\
\hline Age, median year, IQR & 58.0 years $(48.0,65.0)$ \\
\hline \multicolumn{2}{|l|}{ Sex, $n(\%)$} \\
\hline Male & $981(75.2 \%)$ \\
\hline Female & $323(24.8 \%)$ \\
\hline \multicolumn{2}{|l|}{ Surgical hospitals, $n(\%)$} \\
\hline General hospital & $695(53.3 \%)$ \\
\hline Specialist hospital & $600(46.0 \%)$ \\
\hline Unknown & $9(0.7 \%)$ \\
\hline \multicolumn{2}{|l|}{ Tumor location, n(\%) } \\
\hline Gastroesophageal junction & $435(33.4 \%)$ \\
\hline Non-gastroesophageal junction & $865(66.3 \%)$ \\
\hline Unknown & $4(0.3 \%)$ \\
\hline \multicolumn{2}{|l|}{ Histological type, $n(\%)$} \\
\hline Well-differentiated tumors & $301(23.1 \%)$ \\
\hline Poorly differentiated tumors & $842(64.6 \%)$ \\
\hline Signet ring cell cancer & $142(10.9 \%)$ \\
\hline Unknown & $19(1.5 \%)$ \\
\hline \multicolumn{2}{|l|}{ Surgical approach, $n(\%)$} \\
\hline Proximal gastrectomy & 384(29.4\%) \\
\hline Distal gastrectomy & $722(55.4 \%)$ \\
\hline Total gastrectomy & 197(15.1\%) \\
\hline Unknown & $1(0.1 \%)$ \\
\hline \multicolumn{2}{|l|}{ Lymphadenectomy type } \\
\hline D2 lymphadenectomy & $627(48.1 \%)$ \\
\hline D0/D1 lymphadenectomy & $621(47.6 \%)$ \\
\hline Unknown & $56(4.3 \%)$ \\
\hline \multicolumn{2}{|l|}{ T stage, $n(\%)$} \\
\hline $\mathrm{T} 1$ & 100(7.7\%) \\
\hline $\mathrm{T} 2$ & $190(14.6 \%)$ \\
\hline T3 & $586(44.9 \%)$ \\
\hline $\mathrm{T} 4 \mathrm{a}$ & $367(28.1 \%)$ \\
\hline $\mathrm{T} 4 \mathrm{~b}$ & $52(4.0 \%)$ \\
\hline Unknown & $9(0.7 \%)$ \\
\hline \multicolumn{2}{|l|}{ N stage, $n(\%)$} \\
\hline No & $326(25.0 \%)$ \\
\hline N1 & $265(20.3 \%)$ \\
\hline $\mathrm{N} 2$ & $319(24.5 \%)$ \\
\hline N3 & $373(28.6 \%)$ \\
\hline Unknown & $21(1.6 \%)$ \\
\hline \multicolumn{2}{|l|}{ Number of LN dissection } \\
\hline$<15$ & $561(43.0 \%)$ \\
\hline$\geq 15$ & $697(53.5 \%)$ \\
\hline Unknown & $46(3.5 \%)$ \\
\hline
\end{tabular}

Table 1 The clinical pathological characteristics of patents (Continued)

\begin{tabular}{|c|c|}
\hline \multicolumn{2}{|l|}{ AJCC stage, $n(\%)$} \\
\hline IA & $66(5.1 \%)$ \\
\hline IB & $91(7.0 \%)$ \\
\hline$\| \mathrm{A}$ & $174(13.3 \%)$ \\
\hline$\| \mathrm{B}$ & $218(16.7 \%)$ \\
\hline$\| I A$ & $262(20.1 \%)$ \\
\hline$\| I \mathrm{~B}$ & $302(23.2 \%)$ \\
\hline IIIC & $164(12.6 \%)$ \\
\hline Unknown & $27(2.1 \%)$ \\
\hline \multicolumn{2}{|l|}{ Neo-adjuvant chemotherapy } \\
\hline Yes & $63(4.8 \%)$ \\
\hline No & $1177(90.3 \%)$ \\
\hline Unknown & $64(4.9 \%)$ \\
\hline \multicolumn{2}{|l|}{ Adjuvant chemotherapy regimen } \\
\hline No adjuvant chemotherapy & $188(14.4 \%)$ \\
\hline Fluorouracil-based regimens & $970(74.4 \%)$ \\
\hline Other regimens & $44(3.4 \%)$ \\
\hline Unknown & 102(7.8\%) \\
\hline \multicolumn{2}{|l|}{ Adjuvant chemotherapy cycles } \\
\hline No adjuvant chemotherapy & $188(14.4 \%)$ \\
\hline$\leq 3$ cycles & $370(28.4 \%)$ \\
\hline$>3$ cycles & $647(49.6 \%)$ \\
\hline Unknown & $99(7.6 \%)$ \\
\hline \multicolumn{2}{|l|}{ Adjuvant chemotherapy } \\
\hline No & $188(14.4 \%)$ \\
\hline Yes & $1020(78.2 \%)$ \\
\hline Unknown & $96(7.4 \%)$ \\
\hline \multicolumn{2}{|l|}{ Adjuvant radiotherapy } \\
\hline Yes & $26(2.0 \%)$ \\
\hline No & 1209(92.7\%) \\
\hline Unknown & $69(5.3 \%)$ \\
\hline
\end{tabular}

and survival information was January 7th 2015. Recurrence and survival data were obtained from patients' medical records and telephone follow-up.

The recurrence free survival (RFS) was defined as the time from surgery to recurrence or death of any other causes. The overall survival (OS) was defined as the time from operation to death. The recurrence occurred within 2 years after surgery was defined as early recurrence. Recurrence patterns were classified based on the site of the first recurrence. The recurrence inspected within 3 months after the first recurrence was regarded as synchronous recurrence. 


\section{Statistical analysis}

Fifteen clinical relative characteristics were examined as potential risk factors of recurrence. Differences between two groups were assessed by the chi-square or Fisher exact tests. The association of clinical pathological factors with the extent of recurrence was assessed using logistic models. Back-Wald method of multivariate analysis model was used to avoid possible interaction factors for recurrence patterns. Survival curves were analyzed by KaplanMeier method and compared by the log-rank test. All analyses were carried out by SPSS version 22.0. The prognostic powers of covariates were recorded by odds ratios (ORs) and 95\% confidence internals (CIs). All $p$ values $<0.05$ were considered statistically significant.

\section{Results}

\section{Clinical pathological characteristics}

Among the 1304 analyzed patients, the median age was 58.0 years old (IQR 48.0, 65.0). There were 981 males $(75.2 \%)$ and 323 females (24.8\%), with a male-to-female ratio of nearly $3: 1$. The most general tumor location was the antrum $(n=437,33.5 \%)$ and cardiac $(n=387,29.7 \%)$. Most patients $(n=1106,84.8 \%)$ underwent subtotal gastrectomy. The median number of total lymph nodes dissection was 16 (IQR 10.24). And 697 (53.5\%) patients had lymph nodes dissection no less than 15 . Patients had more lymph nodes harvest in specialist hospitals than in general hospitals (median number of lymph nodes harvest 19 [IQR 13.27] vs. 13 [IQR 8.22]). And the specialist hospitals had higher D2 lymphadenectomy rate than general hospitals $(63.0$ vs. $39.0 \%, p<0.001)$. The median number of positive lymph nodes harvest was 3 (IQR 0.7). A majority of patients ( $n=1195,91.6 \%)$ suffered advanced gastric cancer. Only 63 patients (4.8\%) experienced neo-adjuvant chemotherapy, and nobody received neo-adjuvant radiotherapy. A large subset of patients $(n=1020,78.2 \%)$ received adjuvant chemotherapy. Nine hundred seventy patients $(74.4 \%)$ received fluorouracil-based chemotherapy, and 44 patients (3.4\%) received other non-fluorouracil-based chemotherapy. $\mathrm{Al}$ most half of patients $(n=647,49.6 \%)$ received adjuvant chemotherapy over 3 cycles. Only 26 patients $(2.0 \%)$ had adjuvant radiotherapy (Table 1 ).

\section{Recurrence patterns and recurrence-related risk factors Patterns of recurrence and risk factors associated with postoperative recurrence}

The median follow-up was 56.58 months (IQR 30.45, 105.40), and 116 patients (8.7\%) were lost during the follow-up. Seven hundred ninety-three patients (60.8\%) suffered recurrence. And there were 423 patients (32.4\%) with local-regional recurrence, 575 patients (44.3\%) with distant metastasis, and 179 patients (13.7\%) suffering peritoneum implanting (Fig. 2). Four hundred

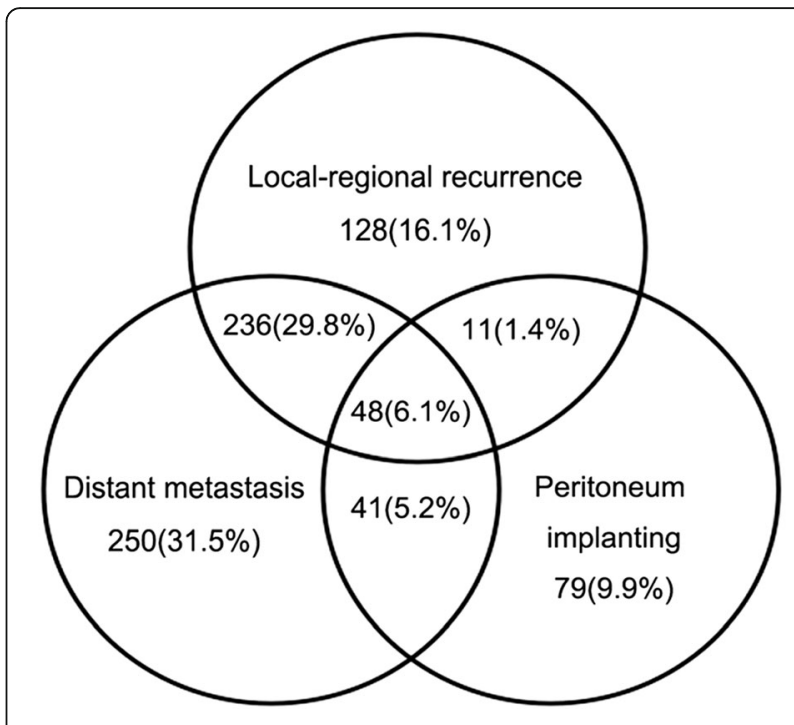

Fig. 2 The recurrence patterns of patients with curative gastrectomy

fifty-seven patients $(57.5 \%)$ experienced single pattern recurrence, while 336 patients (42.5\%) experienced multiple pattern recurrence. The distant lymph node metastasis was more general than distant organ metastasis $(n=392,49.4$ vs. $n=294,37.0 \%)$. The general organs of distant metastasis included liver $(n=154)$, lung $(n=40)$, bone $(n=38)$, pleura $(n=25)$, and subcutaneous nodule metastasis $(n=23)$.

Based on this database, several clinical pathological factors were independent risk factors of recurrence in any site (Table 2). Specially, patients who underwent radical surgery in general hospitals (OR 1.632, 95\% CI 1.238 to 2.151 ) and suffered signet ring cell cancer (OR $1.881,95 \%$ CI 1.108 to 3.193 ) were more likely to experience recurrence. Patients receiving adjuvant radiotherapy (OR $0.259,95 \%$ CI 0.100 to 0.670 ) contributed to lower recurrence risk.

\section{Risk factors of each recurrence pattern}

In exploring risk factors of recurrence patterns, different clinical pathological factors contributed to specific recurrence patterns (Table 3). Receiving operation general hospitals independently risk factors of local-regional recurrence $(\mathrm{OR}=1.724,95 \% \mathrm{CI} 1.312$ to 2.265$)$ and distant metastasis ( $\mathrm{OR}=1.496,95 \%$ CI 1.164 to 1.940$)$. D0/D1 lymphadenectomy increased the risk of localregional recurrence $(\mathrm{OR}=2.272,95 \% \mathrm{CI} 1.734$ to 2.977$)$ and distant metastasis (OR $=1.777,95 \%$ CI 1.369 to 2.307). Patients $>65$ years increased the risk of distant metastasis (OR 1.449, 95\% CI 1.093 to 1.922), but reduced the risk of peritoneum implanting (OR 0.619, 95\% CI 0.403 to 0.984$)$. Patients with factors, including female (OR 1.687, 95\% CI 1.164 to 2.444), signet ring cell cancer (OR 2.627, 95\% CI 1.449 to 4.761 ) were at 
Table 2 The univariate and multivariate analysis of postoperative recurrence

\begin{tabular}{|c|c|c|c|c|c|c|}
\hline \multirow[t]{2}{*}{ Clinical features } & \multicolumn{3}{|c|}{$\underline{\text { Univariate analysis }}$} & \multicolumn{3}{|c|}{ Multivariate analysis } \\
\hline & $\mathrm{OR}$ & $95 \% \mathrm{Cl}$ & $p$ value & $\mathrm{OR}$ & $95 \% \mathrm{Cl}$ & $p$ value \\
\hline \multicolumn{7}{|l|}{ Age } \\
\hline \multicolumn{7}{|l|}{$\leqq 65$} \\
\hline$>65$ & 1.178 & $0.916-1.514$ & 0.201 & NA & NA & NA \\
\hline \multicolumn{7}{|l|}{ Sex } \\
\hline \multicolumn{7}{|l|}{ Male } \\
\hline Female & 0.837 & $0.648-1.080$ & 0.171 & NA & NA & NA \\
\hline \multicolumn{7}{|l|}{ Hospital } \\
\hline \multicolumn{7}{|l|}{ Specialist hospitals } \\
\hline General hospitals & 1.915 & $1.529-2.398$ & $<0.001$ & 1.632 & $1.238-2.151$ & 0.001 \\
\hline \multicolumn{7}{|l|}{ Tumor location } \\
\hline \multicolumn{7}{|l|}{ Non-GEJ } \\
\hline GEJ & 1.683 & $1.319-2.146$ & $<0.001$ & 1.431 & $1.066-1.922$ & 0.017 \\
\hline \multicolumn{7}{|l|}{ AJCC stage } \\
\hline \multicolumn{7}{|l|}{$|-| \mid$} \\
\hline III & 2.608 & $2.070-3.286$ & $<0.001$ & NA & NA & NA \\
\hline \multicolumn{7}{|l|}{ T stage } \\
\hline \multicolumn{7}{|l|}{$\mathrm{T} 1-2$} \\
\hline T3-4 & 2.861 & $2.188-3.741$ & $<0.001$ & 2.332 & $1.675-3.245$ & $<0.001$ \\
\hline \multicolumn{7}{|l|}{ N stage } \\
\hline \multicolumn{7}{|l|}{ NO } \\
\hline$N 1-3$ & 3.397 & $2.617-4.409$ & $<0.001$ & 2.085 & $1.481-2.936$ & $<0.001$ \\
\hline \multicolumn{7}{|l|}{ Positive LN ratio } \\
\hline \multicolumn{7}{|l|}{$\leqq 0.33$} \\
\hline$>0.33$ & 3.577 & $2.759-4.638$ & $<0.001$ & 2.283 & $1.656-3.145$ & $<0.001$ \\
\hline \multicolumn{7}{|l|}{ No. of LN dissection } \\
\hline \multicolumn{7}{|l|}{$<15$} \\
\hline$\geqq 15$ & 0.502 & $0.397-0.635$ & $<0.001$ & NA & NA & NA \\
\hline \multicolumn{7}{|l|}{ Histological type } \\
\hline \multicolumn{7}{|l|}{ Well-differentiated tumors } \\
\hline Poorly differentiated tumors & 0.845 & $0.645-1.107$ & 0.222 & 0.792 & $0.570-1.099$ & 0.162 \\
\hline Signet ring cell cancer & 2.066 & $1.307-3.265$ & 0.002 & 1.881 & $1.108-3.193$ & 0.019 \\
\hline Lymphadenectomy extent & & & & & & \\
\hline D2 & & & & & & \\
\hline D0/D1 & 1.923 & $1.525-2.424$ & $<0.001$ & 2.361 & $1.771-3.147$ & $<0.001$ \\
\hline Neo-adjuvant chemotherapy & & & & & & \\
\hline Yes & & & & & & \\
\hline No & 1.260 & $0.756-2.100$ & 0.374 & NA & NA & NA \\
\hline Adjuvant chemotherapy & & & & & & \\
\hline No & & & & & & \\
\hline Yes & 0.978 & $0.710-1.347$ & 0.892 & NA & NA & NA \\
\hline Adjuvant chemotherapy regime & & & & & & \\
\hline No & & & & & & \\
\hline Fluorouracil-based regimens & 0.947 & $0.687-1.306$ & 0.740 & NA & NA & NA \\
\hline
\end{tabular}


Table 2 The univariate and multivariate analysis of postoperative recurrence (Continued)

\begin{tabular}{|c|c|c|c|c|c|c|}
\hline Other regimens & 2.414 & $1.096-5.315$ & 0.029 & NA & NA & NA \\
\hline \multicolumn{7}{|c|}{ Adjuvant chemotherapy cycles } \\
\hline \multicolumn{7}{|l|}{ No } \\
\hline$\leqq 3$ cycles & 0.754 & $0.527-1.080$ & 0.123 & NA & NA & NA \\
\hline$>3$ cycles & 1.156 & $0.827-1.617$ & 0.396 & NA & NA & NA \\
\hline \multicolumn{7}{|c|}{ Adjuvant radiotherapy } \\
\hline \multicolumn{7}{|l|}{ Yes } \\
\hline No & 2.152 & $0.980-4.725$ & 0.056 & 3.868 & $1.493-10.022$ & 0.005 \\
\hline
\end{tabular}

increased risk of peritoneum implanting. Receiving adjuvant chemotherapy $\leq 3$ cycles could improve the risk of distant metastasis (OR $0.640,95 \%$ CI 0.433 to 0.943 ). Receiving adjuvant radiotherapy could reduce the risk of distant metastasis $(\mathrm{OR}=0.260$, 95\% CI 0.083 to 0.816 ), and potential reduced the risk of local-regional recurrence $(\mathrm{OR}=0.302,95 \% \mathrm{CI} 0.085$ to 1.078$)$.

\section{The analysis for recurrence timing}

During the follow-up, 793 patients experienced recurrence with the overall median RFS of 29.00 months (IQR 12.07, 147.23). There were 554 patients suffering early recurrence, with the median RFS of 10.77 months (IQR 6.00, 15.83). Another 239 patients experienced late recurrence, with the median DFS of 35.83 months (IQR $28.67,50.67)$. Patients with T3-4 (OR $=2.148,95 \% \mathrm{CI}$ 1.537 to 3.001$), \mathrm{N} 1-3 \quad(\mathrm{OR}=1.874,95 \% \mathrm{CI} 1.320$ to $2.660)$, and $\mathrm{LN}+\% \geqq 0.33(\mathrm{OR}=2.024,95 \% \mathrm{CI} 1.531$ to $2.681)$ contributed to the higher early recurrence risk. Having operation in general hospitals has potential to suffer early recurrence $(\mathrm{OR}=1.292,95 \%$ CI 0.995 to 1.676). Having lymph nodes resection no less than 15 $(\mathrm{OR}=0.625,95 \% \mathrm{CI} 0.480$ to 0.815$)$ could reduce the risk of early recurrence.

Among the recurrence patients, the median RFS was only 14.73 months (IQR 7.90, 26.17), and the median RFS varied from recurrence types. The median RFS of single local-regional recurrence, single distant metastasis, single peritoneum implanting, and multiple patterns recurrence was 19.47 months (IQR 10.40, 36.57), 13.67 months (IQR 6.90, 24.27), 14.17 months (IQR $7.73,25.33)$, and 14.33 months (IQR 7.80, 25.70), respectively (Fig. 3a).

\section{The survival outcome of each recurrence type}

There were 668 patients dead during the follow-up, with overall survival of 50.94 months (IQR 26.53, 205.73). Among recurrent patients, the overall survival was as short as 33.63 months (IQR 20.63, 54.87). The median overall survival of single local-regional recurrence, single distant metastasis, single peritoneum implanting, and multiple patterns recurrence was 48.63 months (IQR
24.17, 122.40), 35.70 months (IQR 24.00, 57.80), 29.50 months (IQR 17.90,45.77), and 28.03 months (IQR 18.10, 47.37), respectively (Fig. 3b).

\section{Discussion}

Most studies had noted that curative resection for gastric cancer focused largely on prognosis $[15,16]$. In China, most recurrence pattern data was based on small sample, single-center database [10-12], which could not actually reflect the presence of gastric cancer treatment. And little studies noted recurrence timing and took the surgical hospitals' influence on recurrence into consideration. This study was important, because it firstly aimed to identify the incidence, patterns, and timing of recurrence after curative resection for gastric cancer in a large sample and multiple-center cohort of Chinese patients. The outcomes not only provided Chinese presence of curative resection for gastric cancer but also informed the points, which clinicians should focus during the postoperative follow-up.

As this analysis noted, $60.8 \%$ patients experienced recurrence after curative resection for gastric cancer, which was similar with previous results $[10,12,15]$. The incidence of distant metastasis was comparably high (30-45\%) all over the world [3, 5, 7, 8, 10-12, 17, 18]. Patients in Japan and South Korea suffered lower localregional recurrence compared with China (7-10 vs. 32.4\%) [7-9]. But American patients suffered higher local-regional recurrence in contrast with the Chinese (44.1 vs. $32.4 \%$ ) [3]. However, peritoneum implanting in China was obviously lower than in Japan, South Korea, and USA (13.7 vs. 30-45.9\%) [3, 7, 8, 18, 19]. It might associate with less sensitive imaging examination (even MRI/CT with only $56 \%$ sensitivity for peritoneum implanting) [20], less laparoscopic exploration, and cytological examination of peritoneal lavage fluid. The actual incidence of peritoneum implanting would be higher in China.

Based on this analysis, the difference between surgical hospitals and recurrence was specifically analyzed. Receiving operation in general hospitals contributed to higher recurrence risk and shorter RFS (Fig. 3c, $p<0.001$ ). It partly related less lymph nodes harvest and lower D2 
Table 3 The univariate and multivariate analyses of each recurrence pattern

\begin{tabular}{|c|c|c|c|c|c|c|c|c|c|c|c|c|}
\hline \multirow{3}{*}{ Clinical features } & \multicolumn{4}{|c|}{ Local-regional recurrence } & \multicolumn{4}{|c|}{ Distant metastasis } & \multicolumn{4}{|c|}{ Peritoneum implanting } \\
\hline & \multicolumn{2}{|c|}{ Univariate analysis } & \multicolumn{2}{|c|}{ Multivariate analysis } & \multicolumn{2}{|c|}{ Univariate analysis } & \multicolumn{2}{|c|}{ Multivariate analysis } & \multicolumn{2}{|c|}{ Univariate analysis } & \multicolumn{2}{|c|}{ Multivariate analysis } \\
\hline & $\mathrm{OR}(95 \% \mathrm{Cl})$ & $\overline{p \text { value }}$ & OR $(95 \% \mathrm{Cl})$ & $\overline{p \text { value }}$ & OR $(95 \% \mathrm{Cl})$ & $\overline{p \text { value }}$ & OR $(95 \% \mathrm{Cl})$ & $\overline{p \text { value }}$ & $\mathrm{OR}(95 \% \mathrm{Cl})$ & $\overline{p \text { value }}$ & OR $(95 \% \mathrm{Cl})$ & $p$ value \\
\hline \multicolumn{13}{|l|}{ Age } \\
\hline \multicolumn{13}{|l|}{$\leqq 65$} \\
\hline$>65$ & $\begin{array}{l}1.173 \\
(0.908-1.516)\end{array}$ & 0.223 & $\begin{array}{l}0.744 \\
(0.537-1.030)\end{array}$ & 0.075 & $\begin{array}{l}1.286 \\
(1.008-1.641)\end{array}$ & 0.043 & $\begin{array}{l}1.449 \\
(1.093-1.922)\end{array}$ & 0.010 & $\begin{array}{l}0.552 \\
(0.370-0.822)\end{array}$ & 0.003 & $\begin{array}{l}0.619 \\
(0.403-0.984)\end{array}$ & 0.029 \\
\hline \multicolumn{13}{|l|}{ Sex } \\
\hline \multicolumn{13}{|l|}{ Male } \\
\hline Female & $\begin{array}{l}0.666 \\
(0.502-0.882)\end{array}$ & 0.005 & NA & NA & $\begin{array}{l}0.825 \\
(0.639-1.065)\end{array}$ & 0.140 & NA & NA & $\begin{array}{l}1.861 \\
(1.330-2.603)\end{array}$ & $<0.001$ & $\begin{array}{l}1.687 \\
(1.164-2.444)\end{array}$ & 0.006 \\
\hline \multicolumn{13}{|l|}{ Hospital } \\
\hline \multicolumn{13}{|l|}{ Specialist hospitals } \\
\hline General hospitals & $\begin{array}{l}1.964 \\
(1.545-2.497)\end{array}$ & $<0.001$ & $\begin{array}{l}1.724 \\
(1.312-2.265)\end{array}$ & $<0.001$ & $\begin{array}{l}1.664 \\
(1.332-2.079)\end{array}$ & $<0.001$ & $\begin{array}{l}1.496 \\
(1.164-1.940)\end{array}$ & 0.002 & $\begin{array}{l}1.256 \\
(0.910-1.733)\end{array}$ & 0.165 & NA & NA \\
\hline \multicolumn{13}{|l|}{ Tumor location } \\
\hline \multicolumn{13}{|l|}{ Non-GEJ } \\
\hline GEJ & $\begin{array}{l}1.780 \\
(1.398-2.266)\end{array}$ & $<0.001$ & $\begin{array}{l}1.637 \\
(1.236-2.165)\end{array}$ & 0.001 & $\begin{array}{l}1.226 \\
(0.973-1.546)\end{array}$ & 0.084 & NA & NA & $\begin{array}{l}0.619 \\
(0.431-0.890)\end{array}$ & 0.010 & $\begin{array}{l}0.649 \\
(0.437-0.965)\end{array}$ & 0.033 \\
\hline \multicolumn{13}{|l|}{ AJCC stage } \\
\hline \multicolumn{13}{|l|}{$|-| \mid$} \\
\hline III & $\begin{array}{l}1.455 \\
(1.145-1.850)\end{array}$ & 0.002 & NA & NA & $\begin{array}{l}2.364 \\
(1.879-2.974)\end{array}$ & $<0.001$ & NA & NA & $\begin{array}{l}1.627 \\
(1.165-2.272)\end{array}$ & 0.004 & NA & NA \\
\hline \multicolumn{13}{|l|}{ T stage } \\
\hline \multicolumn{13}{|l|}{$\mathrm{T} 1-2$} \\
\hline T3-4 & $\begin{array}{l}1.752 \\
(1.297-2.368)\end{array}$ & $<0.001$ & $\begin{array}{l}1.495 \\
(1.058-2.113)\end{array}$ & 0.023 & $\begin{array}{l}2.230 \\
(1.684-2.953)\end{array}$ & $<0.001$ & $\begin{array}{l}1.674 \\
(1.210-2.316)\end{array}$ & 0.002 & $\begin{array}{l}3.291 \\
(1.934-5.599)\end{array}$ & $<0.001$ & $\begin{array}{l}3.427 \\
(1.956-6.003)\end{array}$ & $<0.001$ \\
\hline \multicolumn{13}{|l|}{$N$ stage } \\
\hline \multicolumn{13}{|l|}{ No } \\
\hline $\mathrm{N} 1-3$ & $\begin{array}{l}1.913 \\
(1.430-2.558)\end{array}$ & $<0.001$ & NA & NA & $\begin{array}{l}3.179 \\
(2.400-4.212)\end{array}$ & $<0.001$ & $\begin{array}{l}2.354 \\
(1.659-3.341)\end{array}$ & $<0.001$ & $\begin{array}{l}1.808 \\
(1.195-2.736)\end{array}$ & 0.005 & NA & NA \\
\hline \multicolumn{13}{|l|}{ Positive $L N$ ratio } \\
\hline \multicolumn{13}{|l|}{$\leqq 0.33$} \\
\hline$>0.33$ & $\begin{array}{l}2.287 \\
(1.799-2.909)\end{array}$ & $<0.001$ & $\begin{array}{l}1.953 \\
(1.493-2.551)\end{array}$ & $<0.001$ & $\begin{array}{l}2.512 \\
(1.998-3.181)\end{array}$ & $<0.001$ & $\begin{array}{l}1.582 \\
(1.214-2.128)\end{array}$ & 0.001 & $\begin{array}{l}1.509 \\
(1.097-2.078)\end{array}$ & 0.012 & NA & NA \\
\hline
\end{tabular}


Table 3 The univariate and multivariate analyses of each recurrence pattern (Continued)

\begin{tabular}{|c|c|c|c|c|c|c|c|c|c|c|c|c|}
\hline \multicolumn{13}{|l|}{$<15$} \\
\hline$\geqq 15$ & $\begin{array}{l}0.422 \\
(0.332-0.537)\end{array}$ & $<0.001$ & NA & NA & $\begin{array}{l}0.629 \\
(0.502-0.787)\end{array}$ & $<0.001$ & NA & NA & $\begin{array}{l}1.094 \\
(0.794-1.507)\end{array}$ & 0.583 & NA & NA \\
\hline \multicolumn{13}{|l|}{ Histological type } \\
\hline \multicolumn{13}{|l|}{ Well-differentiated tumors } \\
\hline Poorly differentiated tumors & $\begin{array}{l}0.881 \\
(0.666-1.166)\end{array}$ & 0.376 & NA & NA & $\begin{array}{l}0.811 \\
(0.623-1.057)\end{array}$ & 0.121 & NA & NA & $\begin{array}{l}1.589 \\
(1.022-2.472)\end{array}$ & 0.040 & $\begin{array}{l}1.431 \\
(0.889-2.303)\end{array}$ & 0.140 \\
\hline Signet ring cell cancer & $\begin{array}{l}1.308 \\
(0.867-1.823)\end{array}$ & 0.201 & NA & NA & $\begin{array}{l}0.961 \\
(0.645-1.433)\end{array}$ & 0.846 & NA & NA & $\begin{array}{l}3.576 \\
(2.074-6.165)\end{array}$ & $<0.001$ & $\begin{array}{l}2.627 \\
(1.449-4.761)\end{array}$ & 0.001 \\
\hline \multicolumn{13}{|l|}{ Lymphadenectomy extent } \\
\hline \multicolumn{13}{|l|}{ D2 } \\
\hline D0/D1 & $\begin{array}{l}2.442 \\
(1.912-3.119)\end{array}$ & $<0.001$ & $\begin{array}{l}2.272 \\
(1.734-2.977)\end{array}$ & $<0.001$ & $\begin{array}{l}1.595 \\
(1.274-1.996)\end{array}$ & $<0.001$ & $\begin{array}{l}1.777 \\
(1.369-2.307)\end{array}$ & $<0.001$ & $\begin{array}{l}0.933 \\
(0.677-1.286)\end{array}$ & 0.673 & NA & NA \\
\hline \multicolumn{13}{|l|}{ Neo-adjuvant chemotherapy } \\
\hline \multicolumn{13}{|l|}{ Yes } \\
\hline No & $\begin{array}{l}1.285 \\
(0.727-2.272)\end{array}$ & 0.338 & NA & NA & $\begin{array}{l}1.052 \\
(0.630-1.755)\end{array}$ & 0.847 & NA & NA & $\begin{array}{l}1.560 \\
(0.662-3.675)\end{array}$ & 0.309 & NA & NA \\
\hline \multicolumn{13}{|l|}{ Adjuvant chemotherapy } \\
\hline \multicolumn{13}{|l|}{ No } \\
\hline Yes & $\begin{array}{l}0.829 \\
(0.598-1.149)\end{array}$ & 0.261 & NA & NA & $\begin{array}{l}0.909 \\
(0.665-1.242)\end{array}$ & 0.550 & NA & NA & $\begin{array}{l}1.448 \\
(0.883-2.376)\end{array}$ & 0.142 & NA & NA \\
\hline \multicolumn{13}{|l|}{ Adjuvant chemotherapy regimen } \\
\hline \multicolumn{13}{|l|}{ No } \\
\hline Fluorouracil-based regimens & $\begin{array}{l}0.801 \\
(0.577-1.112)\end{array}$ & 0.185 & NA & NA & $\begin{array}{l}0.883 \\
(0.645-1.208)\end{array}$ & 0.436 & NA & NA & $\begin{array}{l}1.417 \\
(0.862-2.329)\end{array}$ & 0.169 & NA & NA \\
\hline Other regimens & $\begin{array}{l}1.649 \\
(0.850-3.199)\end{array}$ & 0.139 & NA & NA & $\begin{array}{l}1.677 \\
(0.862-3.263)\end{array}$ & 0.128 & NA & NA & $\begin{array}{l}1.867 \\
(0.762-4.570)\end{array}$ & 0.172 & NA & NA \\
\hline \multicolumn{13}{|l|}{ Adjuvant chemotherapy cycles } \\
\hline \multicolumn{13}{|l|}{ No } \\
\hline$\leqq 3$ cycles & $\begin{array}{l}0.754 \\
(0.519-1.095)\end{array}$ & 0.138 & NA & NA & $\begin{array}{l}0.707 \\
(0.495-1.008)\end{array}$ & 0.056 & $\begin{array}{l}0.640 \\
(0.433-0.943)\end{array}$ & 0.024 & $\begin{array}{l}1.222 \\
(0.701-2.130)\end{array}$ & 0.479 & NA & NA \\
\hline$>3$ cycles & $\begin{array}{l}0.880 \\
(0.626-1.237)\end{array}$ & 0.463 & NA & NA & $\begin{array}{l}1.055 \\
(0.762-1.461)\end{array}$ & 0.875 & $\begin{array}{l}0.842 \\
(0.588-1.206)\end{array}$ & 0.348 & $\begin{array}{l}1.590 \\
(0.956-2.646)\end{array}$ & 0.074 & NA & NA \\
\hline \multicolumn{13}{|l|}{ Adjuvant radiotherapy } \\
\hline \multicolumn{13}{|l|}{ Yes } \\
\hline No & $\begin{array}{l}2.609 \\
(0.893-7.623)\end{array}$ & 0.080 & $\begin{array}{l}3.316 \\
(0.928-11.848)\end{array}$ & 0.065 & $\begin{array}{l}2.184 \\
(0.911-5.233)\end{array}$ & 0.080 & $\begin{array}{l}3.846 \\
(1.226-12.068)\end{array}$ & 0.021 & $\begin{array}{l}1.229 \\
(0.365-4.138)\end{array}$ & 0.740 & NA & NA \\
\hline
\end{tabular}




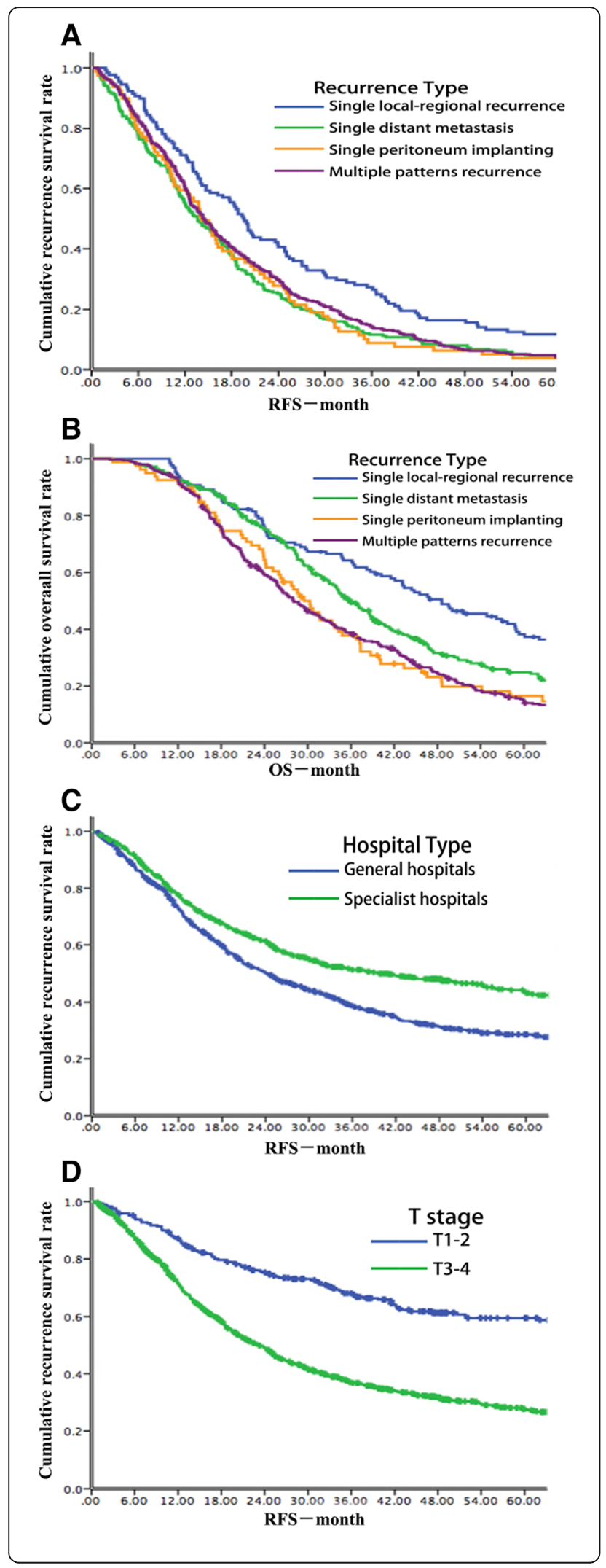

Fig. 3 a The survival curves for RFS of different recurrence patterns. b The survival curves for OS of different recurrence patterns. c The survival curves for RFS of different surgical hospitals. $\mathbf{d}$ The survival curves for RFS of different T status

lymphadenectomy ratio in general hospital. Although the time span of this study was as long as 27 years and D2 lymphadenectomy was just generalized in the past 15 years, specialist hospitals had higher lymph nodes harvest $\geqq 15$ rate D2 lymphadenectomy rate at every period (from Jan. 1986 to Dec. 1999, 40.7 and 34.0\% vs. 19.5 and 13.3\%; from Jan. 2000 to Dec. 2006, 69.0 and $58.1 \%$ vs. 33.2 and 29.9\%; from Jan. 2007 to Sept. 2013, 77.3 and $73.6 \%$ vs. 59.1 and $57.4 \%$ ). It might relate to less volume of curative gastric cancer resection in general hospitals [21] and earlier D2 lymphadenectomy generalization in specialist hospitals. It also referred that standard D2 lymphadenectomy training of clinicians should be continued in China. And increased T stage was the most important independently risk factor of recurrence and with worse RFS (Fig. 3d, $p<0.001$ ). Several studies had noted that postoperative recurrence associated with factors, such as T stage, extent of lymph node invasion, and tumor location $[3,7,10-12,17,19,22-26]$, which were consistent with this study. Based on this database, female and signet ring cell cancer contributed to increased incidence of peritoneum implanting, which reported in previous results $[17,27]$. However, we did not find that lymph node invasion related to peritoneum implanting [22]. The age of patients closely associated with distant metastasis and peritoneum implanting. Patients older than 65 years old had higher risk of distant metastasis and lower risk of peritoneum implanting. In further study, in the group of patients older than 65 years old, less patients were signet ring cell cancer (5.9 vs. $13.0 \%, p<0.001)$ and more patients did not receive adjuvant chemotherapy (22.6 vs. $12.9 \%, p<0.001$ ), comparing with the group of patients no more than 65 years old. It might explain the results. In addition, among patients with signet ring cell cancer, compared with subtotal gastrectomy, patients with total gastrectomy contribute to slightly lower risk of peritoneum implanting (10.0 vs. $15.2 \%)$ and multiple patterns recurrence (23.3 vs. $33.0 \%)$. It might be better to underwent total gastrectomy for signet ring cell cancer patients.

The overall median RFS noting in this analysis was 29.00 months, which was similar to the results in USA [3]. Of note was the further study that the median RFS among patients with recurrence was much shorter, at a little more than 1 year (14.73 months), as the previous study reported $[17,19]$. Single local-regional recurrence occurred later than any other recurrence types and had better overall survival. The overall 3- and 5-year survival rates were 63.8 and $44.8 \%$, respectively, which were higher than 
previous American study (50.9 and 39.3\%) [3]. In further study, the median survival after recurrence was only 13.97 months (IQR 7.03, 24.67), which was consistent with the results of Koizumi W and colleague (13.0 months) [28]. And early recurrence patients had worse survival after recurrence than late recurrence patients (13.50 vs. 16.30 months, $p=0.023)$, which did not show in American analysis [3].

In this study, Chinese patients had more multiple pattern recurrence than patients in America and South Korea (42.5 vs. 33.2 vs. $16.3-27.4 \%)$ [3, 19, 29]. It partly reflected the lack of regular postoperative follow-up in China. A large part of recurrence was early recurrence and 5-year DFS was only 5.2\%. So, that receiving operative examination every 3 months within 2 years and every 6 months within 5 years after surgery were recommended [30, 31], especially for patients with such risk factors.

There were also several limitations in this study. At first, as the patients undergoing surgery in more than 100 hospitals in China, selection bias was unavoidable. Nevertheless, in China, it was general for patients receiving radical resection and adjuvant treatment in different hospitals. Secondly, several other factors previously reported as associated with the recurrence, such as tumor size, vascular tumor thrombus, and Lauren classification did not include this study. Thirdly, the relationship of D2 lymphadenectomy and lymph nodes harvest $\geqq 15$ to recurrence were different, which associated with long time span of surgery and loss of detail surgical records. And then, in this study, we only found had no more than 3 cycles of adjuvant chemotherapy could reduce the recurrence, with statistical significance. More cycles of adjuvant chemotherapy also potential to reduce recurrence. It might associate with different chemotherapy regimens and bias in retrospective study. However, we could not find the association between neo-adjuvant chemotherapy, adjuvant chemotherapy regimens, and recurrence. And although we found that adjuvant radiotherapy could reduce postoperative recurrence and distant metastasis, the results were lower reliability because of small samples. Last but not the least, some patients were lost during follow-up, without information on recurrence or death. All these issues might have led to potential bias in the analysis of recurrence patterns and timing after curative gastric cancer resection.

\section{Conclusions}

In brief, this multiple-center retrospective analysis noted that postoperative recurrence of gastric cancer was common, especially early recurrence. Advanced tumor stage and large tumor burden contributed to increased recurrence risk and worse survival. Enough lymph nodes harvest and standard D2 lymphadenectomy could reduce the postoperative recurrence. Patients might benefit from adjuvant chemotherapy and radiotherapy in China.

\section{Abbreviations \\ AJCC: American Joint Committee on Cancer; Cl: Confidence internal; \\ DFS: Disease-free survival; GEJ: Gastroesophageal junction; IQR: Interquartile range; OR: Odd ratio; OS: Overall survival}

\section{Acknowledgements}

Not applicable.

\section{Funding}

Not applicable.

\section{Availability of data and materials}

Please contact author for data request.

\section{Authors' contributions}

$\mathrm{DL}, \mathrm{ML}$, and $\mathrm{MZ}$ collected clinical data and followed up. LS and ZZ designed this study. DL drafted the manuscript. LS, ML, JL, ZY, and QF revised the manuscript for important intellectual content. All authors read and approved the final manuscript.

\section{Competing interests}

The authors declare that they have no competing interests.

\section{Consent for publication}

Not applicable.

\section{Ethics approval and consent to participate}

Not applicable.

\section{Author details}

'Key Laboratory of Carcinogenesis and Translational Research (Ministry of Education), Department of Gastrointestinal Oncology, Peking University Cancer Hospital and Institute, No. 52, Fucheng Road, Haidian District, Beijing 100142, China. ${ }^{2}$ Division of Epidemiology, The Jockey Club School of Public Health and Primary Care, The Chinese University of Hong Kong, Hong Kong 999077, Hong Kong, China. ${ }^{3}$ Department of Radiation Oncology, Shanghai Cancer Center, Fudan University, Shanghai 200032, China.

Received: 27 August 2016 Accepted: 7 November 2016

Published online: 08 December 2016

\section{Reference}

1. Torre LA, Freddie B, Rebecca L, et al. Global cancer statistics. CA Cancer J Clin. 2015;65:87-108.

2. Chen $W$, Zheng $R$, Zhang $S$, et al. Report of cancer incidence and mortality in China, 2010. Annals of Translational Medicine, 2014, 2(7):61-61

3. Spolverato G, Ejaz A, Kim Y, et al. Rates and patterns of recurrence after curative internt resection for gastric cancer: a United States multi-institutional analysis. J Am Coll Surg. 2014;219(4):664-75.

4. Macdonald JS, Smalley SR, Benedetti J, et al. Chemoradiotherapy after surgery compared with surgery alone for adenocarcinoma of the stomach or gastroesophageal junction. N Engl J Med. 2001;345:725-30.

5. Smalley SR, Benedetti JK, Haller DG, et al. Updated analysis of SWOG-directed intergroup study 0116: a phase III trial of adjuvant radiochemotherapy versus observation after curative gastric cancer resection. J Clin Oncol. 2012;30:2327-33.

6. Isobe Y, Nashimoto A, Akazawa K, et al. Gastric cancer treatment in Japan: 2008 annual report of the JGCA nationwide registry. Gastric Cancer. 2011;14:301-16.4.

7. Eom BW, Yoon H, Ryu KW, et al. Predictors of timing and patterns of recurrence after curative resection for gastric cancer. Dig Surg. 2010;27(6):481-6.

8. Sasako M, Sakuramoto S, Katai H, et al. Five-year outcomes of a randomized phase III trial comparing adjuvant chemotherapy with S-1 versus surgery alone in stage II or III gastric cancer. J Clin Oncol. 2011;29(33):4387-93.

9. Lee J, Lim DH, Kim S, et al. Phase III trial comparing capecitabine plus cisplatin versus capecitabine plus cisplatin with concurrent capecitabine radiotherapy in completely resected gastric cancer with D2 lymph node dissection: the ARTIST trial. J Clin Oncol. 2012;30(3):268-73. 
10. Deng J, Liang $H$, Wang $D$, et al. Investigation of the recurrence patterns of gastric cancer following a curative resection. Surg Today. 2011;41:210-5.

11. Li YM, Zhan WH, Han FH, et al. Analysis of patterns, intervals and risk factors for recurrent gastric cancer. Zhonghua Wai Ke Za Zhi. 2006;44(3):174-6.

12. Kang $W M$, Meng $Q B, Y u J C$, et al. Factors associated with early recurrence after curative surgery for gastric cancer. World J Gastroenterol. 2015;21(19):5934-40.

13. Association J G C. Japanese gastric cancer treatment guidelines 2010 (ver. 3). Gastric Cancer Official Journal of the International Gastric Cancer Association \& the Japanese Gastric Cancer Association, 2011, 14(2):113-123

14. Bosman FT, World Health Organization. International Agency for Research on Cancer. WHO classification of tumors of the digestive system. 4th ed. Lyon: International Agency for Research on Cancer;2010. 417 p. P

15. Ychou M, Boige V, Pignon JP, et al. Perioperative chemotherapy compared with surgery alone for resectable gastro-esophageal adenocarcinoma: an FNCLCC and FFCD multicenter phase III trial. J Clin Oncol. 2011;29:1715-21.

16. Bringeland $\mathrm{EA}$, Wasmuth $\mathrm{HH}$, Johnsen $\mathrm{G}$, et al. Outcomes among patients treated for gastric adenocarcinoma during the last decade. J Surg Oncol. 2013;107:752-7.

17. Wu CW, Lo SS, Shen KH, et al. Incidence and factors associated with recurrence patterns after intended curative surgery for gastric cancer. World J Surg. 2003:27:153-8.

18. Nakagawa M, Kojima K, Inokuchi M, et al. Patterns, timing and risk factors of recurrence of gastric cancer after laparoscopic gastrectomy: reliable results following long-term follow-up. Eur J Surg Oncol. 2014;40(10):1376-82.

19. Yoo CH, Noh SH, Shin DW, et al. Recurrence following curative resection for gastric carcinoma. Br J Surg. 2000;87:236-42.

20. Satoh Y, Ichikawa T, Motosugi U, et al. Diagnosis of peritoneal dissemination: comparison of 18F-FDG PET/CT, diffusion-weighted MRI, and contrast-enhanced MDCT. AJR Am J Roentgenol. 2011;196(2):447-53.

21. Enzinger PC, Benedetti JK, Meyerhardt JA, et al. Impact of hospital volume on recurrence and survival after surgery for gastric cancer. Ann Surg. 2007; 245(3):426-34.

22. Nakanishi Y, Ohara M, Domen H, Shichinohe T, Hirano S, Ishizaka M, et al. Differences in risk factors between patterns of recurrence in patients after curative resection for advanced gastric carcinoma. World J Surg Oncol. 2013;11:98-105.

23. D'Angelica M, Gonen M, Brennan MF, et al. Patterns of initial recurrence in completely resected gastric adenocarcinoma. Ann Surg. 2004;240:808-16.

24. Bilici A, Selcukbiricik F. Prognostic significance of the recurrence pattern and risk factors for recurrence in patients with proximal gastric cancer who underwent curative gastrectomy. Tumour Biol. 2015;36(8):6191-9.

25. Chiang $\mathrm{CY}$, Huang $\mathrm{KH}$, Fang $\mathrm{WL}$, et al. Factors associated with recurrence within 2 years after curative surgery for gastric adenocarcinoma. World J Surg. 2011;35(11):2472-8.

26. Choi JY, Ha TK, Kwon SJ. Clinicopathologic characteristics of gastric cancer patients according to the timing of the recurrence after curative surgery. $J$ Gastric Cancer. 2011:11(1):46-54.

27. Thomassen I, Gestel Y R V, Ramshorst B V, et al. Peritoneal carcinomatosis of gastric origin: a population-based study on incidence, survival and risk factors. International Journal of Cancer Journal International Du Cancer, 2013, 134(3):622-8

28. Koizumi W, Narahara H, Hara T, et al. S-1 plus cisplatin versus S-1 alone for first line treatment of advanced gastric cancer (SPIRITS trial): a phase III trial. Lancet Oncol. 2008:9:215-21.

29. Cho JM, Jang $\mathrm{YJ}$, Kim JH, et al. Pattern, timing and survival in patients with recurrent gastric cancer. Hepatogastroenterology. 2014;61(132):1148-53.

30. Alnoor M, Boys JA, Worrell SG, et al. Timing and pattern of recurrence after gastrectomy for adenocarcinoma. Am Surg. 2015;81(10):1057-60.

31. Ang TL, Fock KM. Clinical epidemiology of gastric cancer. Singapore Med J. 2014:55(12):621-8

\section{Submit your next manuscript to BioMed Central and we will help you at every step:}

- We accept pre-submission inquiries

- Our selector tool helps you to find the most relevant journal

- We provide round the clock customer support

- Convenient online submission

- Thorough peer review

- Inclusion in PubMed and all major indexing services

- Maximum visibility for your research

Submit your manuscript at www.biomedcentral.com/submit
Biomed Central 